\title{
Infrastructure Dependent Wireless Multicast - The effect of Spatial Diversity and Error Correction
}

\author{
Jean Marie Vella \\ Dept. of Communications and Computer Engineering \\ University of Malta \\ Msida, Malta \\ jve10009@um.edu.mt
}

\author{
Saviour Zammit \\ Dept. of Communications and Computer Engineering \\ University of Malta \\ Msida, Malta \\ saviour.zammit@um.edu.mt
}

\begin{abstract}
The use of multiple Access Points (APs) with one AP placed at the middle of a coverage area and the remaining placed at the edge may reduce the Packet Error Rate (PER) experienced by a group of multicast receivers. This paper shows that Spatial Diversity can augment the channel quality experienced especially by those nodes which are located farther from the master AP, i.e. the AP at the middle, however this study also demonstrates the need for error correction scheme. The aim of this analysis is to propose a means of enhancing the infrastructure end of an IEEE 802.11n Wireless Local Area Network (WLAN), such that multicast data can be delivered reliably in order to guarantee that the received video has an adequate Peak Signal to Noise Ratio (PSNR), but with the constraint that the Medium Access Control (MAC) and the Physical (PHY) layer of the receivers are not modified, hence a legacy IEEE 802.11n node may join the multicast group and experience good Quality of Service.
\end{abstract}

Index Terms-wireless multicast; video streaming; cooperative relaying; packet redundancy; spatial diversity

\section{INTRODUCTION}

Multicasting is a bandwidth-efficient means of transmitting data to a group of receivers since it takes advantage of the broadcasting nature of the wireless medium, by sending one data packet addressed to and received by a group of nodes. Data transmitted over a wireless channel may be lost or received corrupted because of the random nature of this medium. Hence, the IEEE 802.11 standard [1] uses Carrier Sense Multiple Access with Collision Avoidance (CSMA/CA), Request-to-Send (RTS), Clear-To-Send (CTS) and Acknowledgement (ACK) in order to increase the reliability of wireless transmission. CSMA/CA constraints a transmitter to first sense the channel and if it hears an on-going transmission it must back-off. The RTS and CTS are transmitted by the source node and destination node respectively, in order to guarantee that the receiver is not participating in another transmission and also to avoid the hidden node problem, whereas ACKs are send to the source whenever a transmission is successful. Hence the lack of ACK is a sign that a packet was

The research work disclosed in this publication is funded by the Strategic Educational Pathways Scholarship (Malta). The scholarship is part-financed by the European Union European Social Fund (ESF) under Operational Programme II Cohesion Policy 2007-2013, Empowering People for More Jobs and a Better Quality of Life

978-1-4673-2017-7/12/\$31.00 @2012 IEEE not received successfully and therefore a retransmission is required. But since multicast involves the transmission of a data packet to a group receiver and the source cannot receive feedback from multiple nodes simultaneously, the IEEE 802.11 standard does not use RTS/CTS and ACK for multicast transmission. Hence, this means of transmission over IEEE 802.11 wireless local area networks (WLANs) has the disadvantage that it suffers from lack of reliability.

Since ACK are not used, data rate adaptation and contention window adaptation are eliminated. This means that the source selects one modulation and coding scheme (MCS), which the standard states should be one of the basic service set (BSS) basic rate set, to transmit the entire multicast data. Since multicast over IEEE 802.11 WLAN networks does not employ any reliability techniques, the most robust MCS is selected. This results in the "performance-anomaly" problem [2]. The lack of contention window adaptation means that a multicast source does not backoff when the transmission is not successful. However, other unicast sources do backoff in such a case. Hence multicast is unfair with unicast transmission [3].

However, the throughput optimization that can be obtained via multicast has driven the research community to design reliable multicasting technique. Moreover, IEEE TGaa has been formed to improve multicast over WLANs. This is beneficial to and stimulated by the proliferation of hand-held devices with IEEE 802.11 transceivers and ubiquitous WiFi spots. Hence, this paper is part of a study that aims to augment video streaming over IEEE 802.11n WLANs using multicast. Special interest is paid to applications which involve massgathering events such as concerts, or football matches, where users can receive live footage of the on-going show or match on their handheld devices without having to depend on big screens. The study has already empirically and analytically showed that the infrastructure can be modified to take advantage of spatial diversity by placing additional transmit antennas at the edge of the coverage area considered [4], [5], [6]. However, using one AP with multiple antennas does not offer error correction techniques. Therefore in this paper a cooperative system enhancing the infrastructure by deploying additional APs at the edge of a coverage area is presented.

The paper is organized as follows. The following section presents a brief review of the literature considered with multicast over WLANs. Section III describes the InfrastructureDependent Wireless Multicast concept and how it differs from 
other cooperative schemes. The methodology employed to test the use of multiple APs is included in Section IV, while the results and a discussion of the results form Section V. The paper is then concluded by analyzing possible future directions.

\section{RELATED WORK}

A thorough survey of multicast over wireless access networks is presented in [7]. This survey shows that various techniques have been proposed to solve the lack of reliability including link-layer reliability such as promiscuous reception of unicast transmission, Leader-Based Protocols or by polling some or all of the multicast group member requesting ACK. However, a brief description of Cooperative Relaying over IEEE 802.11 WLANs is presented here.

One of the common features of cooperative relaying is the use of multicast group members as relays. Hence the AP transmits the data during the first portion of a time slot and then the relays are responsible of transmitting the data to the nodes located farther away from the AP. Alay et al. [8], [9] state that in this manner, the AP can use a higher Physical Layer (PHY) data rate than that necessary if it aims to transmit its multicast data to the entire group members which may be located anywhere within the coverage area. However multicast group members may be battery-powered devices, therefore the receivers which act as relays use some of their energy to service nodes which are located farther away. Alay et al. [8] argue that the idea behind such a concept is that since relays may be mobile, the nodes acting as relays will not be the same nodes during the entire stream. But this may not be necessary especially if one considers the scenario of a stadium with a football match where the supporters will spend most of the entire match located in the same position. The work produced by Alay et al. developed from the use of relays with omnidirectional antennas [8] to relays with directional antennas [9] and then to the employment of Random Distributed Space Time Codes [10] such that the relays can act as a virtual antenna array. In their study Alay et al. also stated that in case of video streaming, scalable encoded video may be used such that the AP transmits both the base layer and the enhancement layers but the relays may transmit only the base layer [8], [9], [10]. The disadvantage of such a scheme is that nodes which are located farther from the AP receive a lower video quality than those nodes located close to the AP. However, this is not appropriate since both types of receivers may have paid the same fee in order to receive the video stream.

Yoon et al. [11] also used relays which are part of the multicast group members to forward data, however they allow the nodes which receive the data from the relays to select the relays from which they receive the data. Moreover, multiple number of channels are used, such that each relay may choose an unused channel so that relays can transmit simultaneously the multicast data.

Instead of retransmitting each packet, Fan et al. [12] opted to use network coded packets in which each combined packet is a XORing of lost packets. In such a manner a destination node may recover one lost packet out of each combined packet given that it had previously received the other packets forming part of the combined packet. The recovery process uses both transmission from a relay node and the source, where the source is used to transmit network coded packets which were not received successfully by the relay node. This scheme has the disadvantage that it requires feedback from all the nodes such that for every $\mathrm{N}$ packets transmitted from the source, the relay and the source know which packets were received by each destination. It is not advantageous to combine multicast and ACK feedback over an IEEE 802.11 WLAN since both the source and the relay cannot receive feedback from all the receivers simultaneously. More if unicast was to be used to transmit the feedback to the source and the relay, the delay related to the feedback process is linear with the number of multicast receivers. Therefore, this is not an appropriate scheme for the mass-gathering events considered in this paper.

\section{INFRASTRUCTURE-DEPENDENT WIRELESS MULTICAST}

The goal of infrastructure-dependent wireless multicast (IDWM) is to offer a ubiquitous resilient connection to the entire multicast group over a WLAN, requiring no hardware changes at the receiver end and implementing all the changes at the infrastructure end, so that any IEEE $802.11 \mathrm{n}$ device can connect to such a system. In a standard WLAN, it is not possible for all the receivers to be located close to the AP which transmits the multicast data as shown in Fig. 1. However, this means that the nodes located farther from the AP will suffer from attenuation and hence a higher PER, than those nodes located near the AP. Through the results presented in [4] and [5], it has also been shown that the PER experienced by a node may be impacted by the hardware and/or driver that the receiving node uses. One other point that can be noted from Fig. 1 is if one considers such a WLAN to be implemented for a stadium during a concert or live football match, where people are usually standing close together side by side, there will be a larger number of receivers located farther away from the AP than close to the AP. Hence, it is important that the effect of location-dependent losses is reduced. Therefore IDWM is proposed, which places additional transmitting sources at the extremities of the coverage area, where the effect of attenuation will be mostly felt.

This concept has already been tested using an IEEE 802.11n testbed with an AP having multiple antennas, placing an antenna at each end of the coverage area [4], [5]. But in this paper space diversity is created using multiple APs instead of multiple antennas creating a WLAN as shown in Fig. 2. The advantage of deploying multiple APs instead of one AP with multiple antennas is the use of the full transmit power by each $\mathrm{AP}$, whereas if the use of two transmit antennas placing them at opposite end is assumed, each location experiences a $3 \mathrm{~dB}$ loss in power which increases the packet error rate (PER) of those nodes already located close to the original transmitting source as shown in [6]. Moreover, the use of multiple APs allows one to employ any number of APs whereas using an AP with multiple antennas will be limited by the number of antennas at the AP. Also one can control what each AP transmits whereas when an AP with multiple antennas is employed, the transmitted signals from each antenna are the same but spatially expanded. However it has already been shown both empirically and analytically [4] [5] [6] that the use of transmit antennas placed at opposite ends of the coverage area, beside the transmitting source at the middle, can be beneficial for multicast over WLANs reducing the maximum PER reported. 
The IEEE 802.11 Standard [1] states that each receiver can only be associated and authenticated with one AP. Hence this also means that it will receive only the data packets having the BSSID of that AP. Therefore, if multiple APs are deployed, each having their own BSSID, a receiver must first disassociate from the current AP and associate and authenticate with another AP, before it can receive data from the new AP, which causes a delay during which multicast data packets cannot be received. Hence a Master AP and Slave APs concept is presented, where these devices act as if they are one entity. Thus when the receiver scans for an AP it will detect only one and as far as the receiver is aware it will connect to one BSS. In order to achieve this, the Master AP must handle the transmission and reception of control frames that enable association and authentication. This is similar to the Divert concept proposed by Miu et al. [13]. Also the Master AP is responsible of beacon frame transmission and probe responses. The Slave AP only feature when there is a multicast transmission, although one may use them for unicast retransmission as proposed by Miu et al. in [13] or to increase reliability over the uplink since a transmitted packet on the uplink can now be received by either the Master AP or any of the Slave APs without forcing the IEEE 802.11n transceivers to be able to join more than one BSS as proposed by Zhu et al. [14]. However, in this study they are designed to transmit only groupcast-addressed data. In order for the receivers to receive the data from the Slave APs too, MAC address spoofing must be performed so that the MAC address of the Slave APs can be set to be the same as that of the Master AP. Also the Master and Slave APs must operate on the same frequency channel. Hence in order not to interfere with each other's transmission, the Master and Slave APs operate in orthogonal time slots. However, since the slave APs are placed at the edge of the coverage area, opposite Slave APs may transmit at the same time slot. This may cause interference at the nodes in the middle but these are experiencing good channel quality with the Master AP and hence do not depend on the Slave APs.

It is assumed that there is a reliable connection between the Master AP and each Slave AP, hence the packets that are scheduled to be transmitted by the Slave APs are reliably delivered to the Slave AP. This can be easily implemented using either a wired link between each AP or else using highly directional antennas between the Master AP and the Slave APs for an out-of-band transmission of the data packets. In this paper, it was assumed that there is a wired network connecting each AP together and to a server. The server controls which packets are delivered by the Master AP and/or the Slave APs. Therefore an application layer implementation was used to switch transmission between the two types of AP.

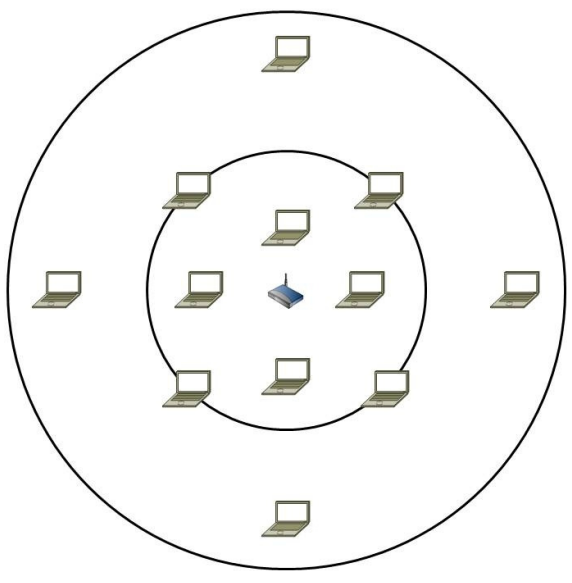

Fig. 1. Basic Service Set

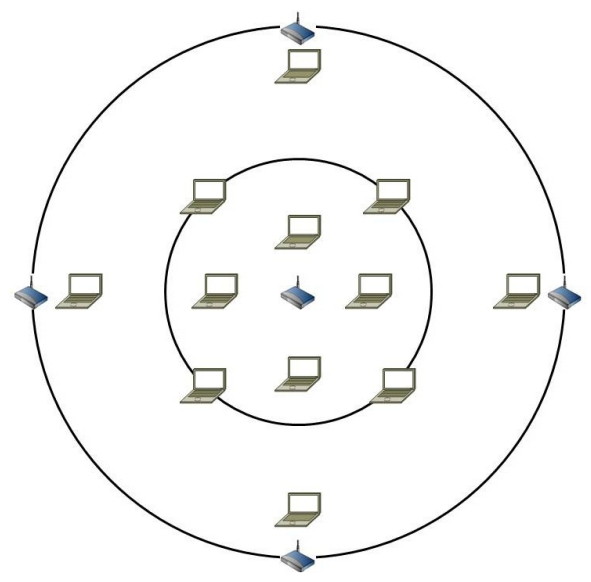

Fig. 2. Infrastructure Dependent Wireless Multicast

The differences between the IDWM concept and the previous relaying cooperative proposals are:

1. The Slave APs are part of the infrastructure and hence are not multicast group members which, for the application under consideration i.e. multicasting live footage in a stadium during a football match or a concert, would be battery powered. This differs from the proposal by Alay et al. [8], [9] where the relays are multicast group members. As a result there is no need to perform relay selection [11].

2. The Slave APs are placed at the edge of the coverage area and not in the middle of the coverage area. IDWM has this flexibility since the Slave APs are part of the infrastructure and therefore can be connected via a wired connection to a server just like the Master AP. Therefore, as one may assume that the connection between the server and the Master AP is a reliable one, similarly the connection between the server and the Slave APs is robust. However, when the relays are some of the nodes of the multicast group, they must be located at a position which ensures that a good channel quality is experienced so that they can receive and relay most of the packets transmitted by the AP.

3. The Slave APs differ from infrastructure-based fixed relays because the receivers do not use physical layer 
techniques to combine the signal from the source with the signal from the Slave APs as is the case when amplify-and-forward or decode-and-forward relays are used [15], [16]. Moreover the Slave APs do not transmit simultaneously using techniques such as Randomized Distributed Space Time Codes [10] to create a virtual array or synchronization of the OFDM symbols as proposed in SourceSync [17] again allowing all-ready available IEEE $802.11 \mathrm{n}$ receivers to connect and receive the video multicast stream.

4. The multicast group members are not aware that the infrastructure consists of both Master and Slave APs. One of the main constraints of the IDWM concept is that the receivers do not require any changes except at the Application Layer in order to receive data from the Master and Slave APs, hence the receivers do not need to delay the data received from the Master AP in order to combine it with that from the Slave AP [16].

5. The IDWM is not a two-hop cooperative system. The data from the Master AP may be received by all nodes. The Slave APs are not used to increase the coverage area, hence are not responsible to transmit the data to those nodes which are out of range from the Master AP but the aim is to overcome losses and increase resilience. Each multicast group member can receive data from both the Master AP and the Slave AP. In fact a node which is out of range from the Master AP and hence cannot perform association and authentication cannot receive data from neither the Master AP nor the Slave AP.

6. While this paper is proposing packet repetition as a redundancy technique, Slave APs can be used to transmit parity packets, coded using RS codes, or transmit innovative network coded packets; techniques which shall be studied in the future.

7. Even though both Divert and IDWM use a combination of APs transmitting over the same frequency with one AP i.e. the Master AP or as referred to by Miu et al. [13] Primary AP, handling the association and authentication, the IDWM constantly uses the Slave APs unlike the Divert which uses feedback from their unicast receiver in order to switch the transmission to another AP. Since multicast considers a number of receivers, switching to one AP may harm the transmission of another receiver which was experiencing a good channel quality from the Master AP. Hence the Master AP and Slave APs must work in harmony in order to ensure that the entire multicast group experiences a low PER. This means that the IDWM proposal is adequate for multicast applications unlike Divert which may be applied only for unicast applications since it requires feedback from the receiver. IDWM is also simpler to implement because the Master and Slave APs do not need to sniff and know which receivers are close to their location or not.

\section{METHODOLOGY}

As the transmission from each interfering AP is orthogonal in time so that the APs do not act as interfering sources to each other, a half a sector is simulated, which can show the effect of a Master AP and a Slave AP on the nodes in this area. The nodes considered are located as shown in Fig. 3 and Fig. 4 which shows that a coverage area of $60 \mathrm{~m}$ is considered. The nodes considered in this half a sector are placed at a distance of $20 \mathrm{~m}, 30 \mathrm{~m}, 40 \mathrm{~m}$ and $60 \mathrm{~m}$ from the Master AP and $20 \mathrm{~m}, 30 \mathrm{~m}$ and $40 \mathrm{~m}$ from the location where a Slave AP in this sector would be positioned. In the simulations, independent of whether a single AP or a Master and Slave AP configuration is considered all 28 nodes are employed in the study. Fig. 3 clearly shows that in a mass-gathering event such as a concert there will be more receivers located farther from the Master AP then close to it because the spatial area is larger the farther away a node is. Hence it is pertinent to reduce the effect of attenuation.

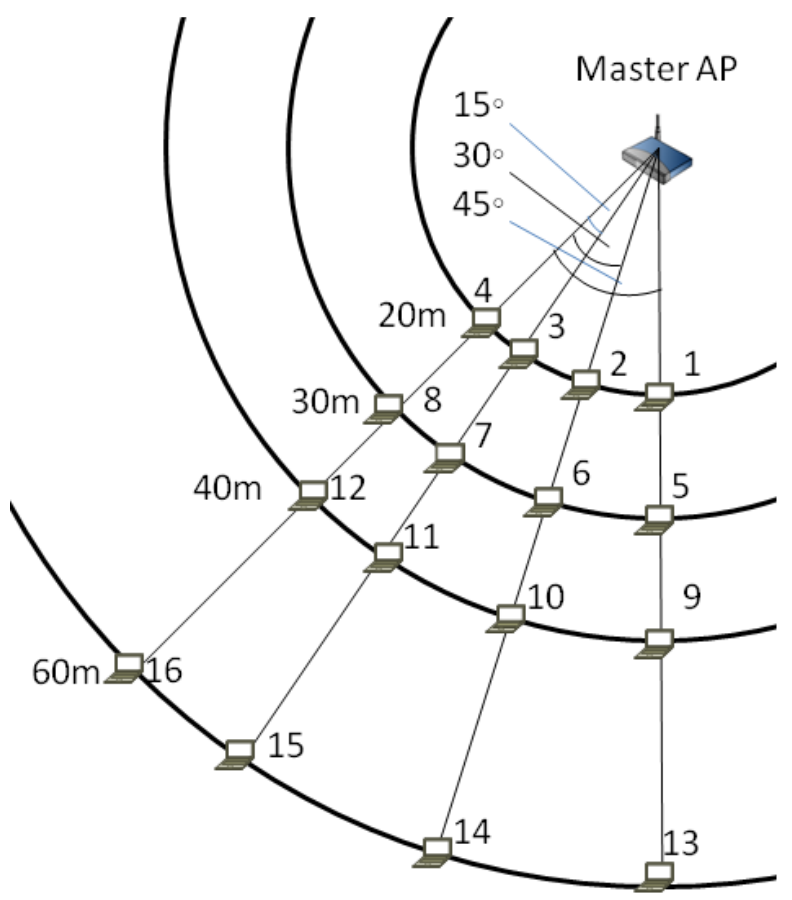

Fig. 3. Distribution of nodes relative to Master AP 


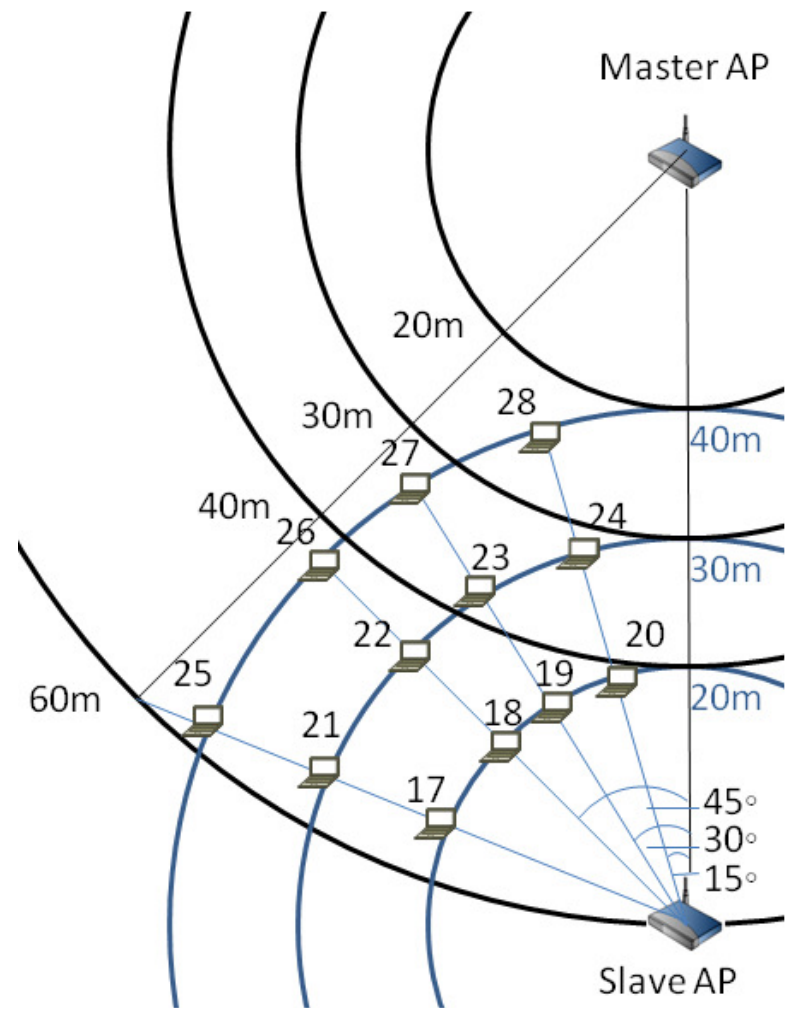

Fig. 4. Distibution of nodes relative to Slave AP

Since the study is aimed for video multicast over IEEE 802.11n WLAN, a PHY abstraction is first performed, as ns3.13 [18], the software used to simulate our network does not yet include the IEEE 802.11n PHY. In order to abstract the PHY layer, a Matlab script [19] which generates IEEE 802.11n TGn channels was used. In this case since the application considered focuses on an outdoor WLAN, channel model F [20] was used. However, the Matlab script was modified such that even at a distance of $60 \mathrm{~m}$ from a transmitting source a line-ofsight (LOS) channel is experienced, because the original channel model $\mathrm{F}$ has a breakpoint at $30 \mathrm{~m}$, hence at a larger distance then the breakpoint a non-LOS channel is assumed, but this is not the case for the scenario considered. Therefore, for every node considered, a channel was created considering a LOS between itself and the Master AP and another channel with LOS from the Slave AP. It is assumed that the channels are SISO. This is justified by the fact that a single antenna at the Master and Slave APs is the worst possible case since it has already been shown that using multiple antennas at the transmitter reduces the PER experienced even if Spatial Expansion is employed [4], [5]. Moreover, the IEEE 802.11n standard [1] does not state that each receiver should have multiple antennas. Hence several single-stream transceivers are available on the market. Therefore as it has already been stated in [5], a multicast solution should allow single stream receivers to join the multicast group and receive the data. Another assumption that was adopted is that the channel remains constant during a packet transmission. Therefore, since each run assumed 50,000 packets in order to extract its simplified Gilbert model parameters, 50,000 channel instances were generated.
TABLE I PHYSICAL LAYER PARAMETERS

\begin{tabular}{|c|l|}
\hline Carrier frequency & $5.25 \mathrm{GHz}$ \\
\hline Signal Bandwidth & $20.0 \mathrm{MHz}$ \\
\hline Data Subcarriers & 52 \\
\hline Pilot Subcarriers & 4 \\
\hline Preamble Format & Greenfield Mode \\
\hline Guard Interval & $800 \mathrm{n} \mathrm{sec}$. \\
\hline Transmit Power & $20 \mathrm{dBm}$ \\
\hline Noise Figure & $10 \mathrm{~dB}$ \\
\hline Antenna Gain & $0.0 \mathrm{dBi}$ \\
\hline Antenna Pattern & Omni-Directional \\
\hline
\end{tabular}

Another Matlab script was then used to output the simplified Gilbert Model parameters by following a procedure similar to that used by Xia et al. [21], which was described in detail in [6]. Hence a brief description is presented here. For each channel instance the effective SNR was evaluated using the same approach proposed by Bjerk et al. in [22]. Then for the MCS considered the Matlab script calculates the bit error rate, the probability of receiving an OFDM symbol correctly and then the packet error rate. The mathematical equations used in order to extract the packet error rate are discussed in [6]. Then a "weighted coin toss" [22] is performed to decide whether the packet considered is in error or not. Considering the entire 50,000 packet stream, the simplified Gilbert Model parameters were evaluated i.e. the probability that a channel goes from a bad state to a good state and vice versa. The simplified Gilbert Model [25], which was also used by Ismail et al. [26], receives a packet successfully when the state of the channel is Good and drops a packet when the state is Bad. For each location and each MCS considered, this process was repeated 10 times and an average was then obtained. Gilbert parameters were extracted considering PSDU payloads of size 100 bytes up to 1000 bytes incremented in steps of 100bytes. The simplified Gilbert Model were then included in ns3. The ns3 was modified to incorporate the $802.11 \mathrm{n}$ Greenfield preamble durations and the MCS 5, 6, 7. The parameters of the considered MCS are tabulated in Table II.

Since the application under consideration is multicasting live footage of events such as a concert or a football match i.e. fast changing video, the video that was transmitted during the simulations is football [23] in CIF format encoded at 30 frames per second using the H.264 reference software JM18.2 employing the Baseline profile. The video was encoded using the slice mode ensuring that a slice is not larger than 926 bytes. One group of pictures consists of 16 frames, the first being an I-frame and the rest being P-frames. Each ns3 simulation run transmitted 2,097 packets and was repeated for 25 times in order to simulate more than 50,000 packets. Since the JM18.2 decoder does not support slice level errors, the decoding was performed using ffmpeg-0.11 [24]. The ffmpeg-0.11 was modified such that it displays the frame number of each decoded frame. Missing frames were then replaced using the previous frame.

In this paper two methodologies are studied. First an analysis is performed of the PER and the respective PSNR at each node if the Master and Slave AP were used to increase redundancy by spatial diversity only. Hence, ns3 is used to simulate the effect of transmitting a packet from the Master AP and then the next packet is transmitted from the Slave AP, therefore each packet is only transmitted once either from the 
TABLE II MCS PARAMETERS [1]

\begin{tabular}{|c|c|c|c|}
\hline MCS index & Modulation & Coding Rate & Data Rate (Mbps) \\
\hline 5 & 64-QAM & $2 / 3$ & 52.0 \\
\hline 6 & 64-QAM & $3 / 4$ & 58.5 \\
\hline 7 & 64-QAM & $5 / 6$ & 65.0 \\
\hline
\end{tabular}

Master AP or from the Slave AP where the transmission from the Master AP and from the Slave AP alternates. This is compared to the resulting PER and PSNR if only the Master AP is used to transmit each packet once. Then Spatial Diversity is combined with packet redundancy, a simple error correction scheme which does not require feedback from the nodes. Therefore each packet is first transmitted from the Master AP and then it is retransmitted from the Slave AP. This is compared to the effect of retransmitting every packet proactively from the Master AP. In order to know which packets were received and which were lost, a 6 byte sequence number is added to the application layer payload. This also allows the receivers to know if a packet was already received from the Master AP and hence, discard the packet from the Slave AP.

\section{RESULTS}

\section{A. Spatial Diversity Only}

Table III shows the PER and the PSNR experienced by the 28 nodes considered when each packet is transmitted once from the Master AP and when the packets are transmitted once alternating between the Master AP and Slave AP using mcs-5. With respect to the PSNR, two parameters are shown; Count and PSNR. Count gives the number of times out of the 25 simulation runs that a node did not achieve exactly the same copy of the decoded video. Whereas PSNR is the average PSNR considering only those simulation-runs where the decoded video was not exactly a copy of the decoded video if all packets transmitted were received.

One can notice that the nodes located farthest from the AP are the ones which benefit the most from the Spatial Diversity. This is similar to the outcome which was observed when multiple antennas were used instead of multiple APs as demonstrated in [4], [5], [6]. In fact nodes 13, 14 and 15 experience an average PER improvement of $50 \%$ when the Slave AP is used. On the other hand Node 16 which is located at $45.9 \mathrm{~m}$ away from the Slave AP experiences an improvement of $35 \%$. However despite the benefit that Spatial Diversity imposed on the nodes located farther from the AP, one can note that it was not enough to obtain a PSNR of greater than at least $30 \mathrm{~dB}$ [27]. The nodes which experienced the largest increase in PER when the Slave AP was used are Node 3, 4 and 8. The three nodes were experiencing a good channel quality when the transmission was from the Master AP only but when half of the packets were transmitted from the Slave AP which is located farther away, their PER increased. Even though one may state that the use of the Slave AP without packet redundancy decreased the maximum PER and moreover 17 nodes out of the entire 28 nodes experienced an improvement in PER, while 4 nodes maintained approximately the same channel quality i.e. nodes 1, 5, 6 and 12, the improvement is not enough to state
TABLE III. SPATIAL DIVERSITY ONLY - MCS-5

\begin{tabular}{|c|c|c|c|c|c|c|}
\hline \multicolumn{2}{|c|}{ Master AP only } & \multicolumn{3}{c|}{ Master AP and Slave AP } \\
alternating
\end{tabular}

that this multicast group will experience good video quality. Hence Spatial Diversity is not enough to multicast video over WLAN.

\section{B. Spatial Diversity and Packet Redundancy}

One can observe a significant improvement from Table IV when packet diversity is combined with spatial diversity. In fact the maximum PER obtained is $0.56 \%$ whereas without the use of error correction, nodes $13,14,15$, and 16 obtain an average PER of $10.62 \%$ which leads to a low average PSNR of $23.47 \mathrm{~dB}$. It can be noticed also that unlike the results presented in [6], where the nodes close to the main transmitting source location obtained an increase in PER when two transmit antennas were used with one of the antennas placed at the edge of the coverage, nodes 1,2,3, and 4 maintained an excellent channel quality because the master AP is still using the same transmit power and transmitting all of the multicast data packets. The increase in PER observed in [6] resulted from a $3 \mathrm{~dB}$ decrease in transmit power because the total transmit power is halved between each transmit antenna.

With respect to the use of MCS-6, the maximum PER decreased from $17.69 \%$ to $1.72 \%$, the latter PER being observed by node 16. Its corresponding PSNR was that of $31.05 \mathrm{~dB}$ which is lower than the desired 35dB [28] for H.264. However, one must point that this node will receive transmission from two Slave APs, because it is located at the border of the sector covered by a Slave AP and hence, the final PER would be lower than the one obtained. The remaining 27 
TABLE IV. PACKET REDUNDANCY - MCS-5

\begin{tabular}{|c|c|c|c|c|c|c|}
\hline & \multicolumn{3}{|c|}{ Master AP only } & \multicolumn{3}{|c|}{$\begin{array}{c}\text { Master AP and Slave AP } \\
\text { alternating }\end{array}$} \\
\hline Node & $\begin{array}{c}\text { PER } \\
(\%)\end{array}$ & Count & $\begin{array}{c}\text { PSNR } \\
\text { (dB) }\end{array}$ & $\begin{array}{c}\text { PER } \\
(\%)\end{array}$ & Count & $\begin{array}{c}\text { PSNR } \\
\text { (dB) }\end{array}$ \\
\hline 1 & 0.00 & 0 & & 0.00 & 0 & \\
\hline 2 & 0.00 & 0 & & 0.00 & 0 & \\
\hline 3 & 0.00 & 0 & & 0.00 & 0 & \\
\hline 4 & 0.00 & 0 & & 0.00 & 0 & \\
\hline 5 & 0.07 & 20 & 45.12 & 0.00 & 0 & \\
\hline 6 & 0.03 & 15 & 47.71 & 0.00 & 0 & \\
\hline 7 & 0.07 & 19 & 43.19 & 0.01 & 2 & 43.37 \\
\hline 8 & 0.06 & 19 & 44.90 & 0.01 & 4 & 45.45 \\
\hline 9 & 0.76 & 25 & 34.39 & 0.00 & 0 & \\
\hline 10 & 0.78 & 25 & 34.57 & 0.00 & 0 & \\
\hline 11 & 0.75 & 25 & 34.67 & 0.00 & 1 & 43.06 \\
\hline 12 & 0.58 & 25 & 35.67 & 0.05 & 12 & 44.67 \\
\hline 13 & 10.31 & 25 & 23.55 & 0.00 & 0 & \\
\hline 14 & 11.15 & 25 & 23.42 & 0.00 & 0 & \\
\hline 15 & 10.87 & 25 & 23.28 & 0.02 & 7 & 44.31 \\
\hline 16 & 10.22 & 25 & 23.62 & 0.56 & 25 & 36.95 \\
\hline 17 & 6.58 & 25 & 25.30 & 0.00 & 0 & \\
\hline 18 & 3.36 & 25 & 27.89 & 0.00 & 0 & \\
\hline 19 & 2.34 & 25 & 29.19 & 0.00 & 0 & \\
\hline 20 & 0.70 & 25 & 34.65 & 0.00 & 0 & \\
\hline 21 & 7.33 & 25 & 24.814 & 0.01 & 5 & 50.52 \\
\hline 22 & 2.43 & 25 & 29.17 & 0.02 & 1 & 55.28 \\
\hline 23 & 0.37 & 25 & 37.58 & 0.00 & 0 & \\
\hline 24 & 0.14 & 23 & 43.27 & 0.00 & 0 & \\
\hline 25 & 8.59 & 25 & 24.14 & 0.13 & 19 & 43.57 \\
\hline 26 & 1.45 & 25 & 31.58 & 0.05 & 11 & 45.08 \\
\hline 27 & 0.18 & 25 & 42.23 & 0.02 & 4 & 45.93 \\
\hline 28 & 0.03 & 11 & 46.43 & 0.00 & 0 & \\
\hline
\end{tabular}

nodes all obtained a PER lower than $0.35 \%$ with a PSNR of at least $39 \mathrm{~dB}$.

The results for the least robust MCS that transmits one spatial stream over IEEE $802.11 \mathrm{n}$ are tabulated in Table V. Nodes $4,8,12$, and 16 are at the border of the sector, hence they will receive transmission from two Slave APs. Therefore, their channel experience will be better than the one indicated in Table V. Moreover, if the coverage area of the Slave AP is the same as the Master AP i.e. $60 \mathrm{~m}$ then even nodes 21, 25 and 26 will experience transmission from two Slave APs. However it is better if the transmission from one Slave AP is considered because it allows one to determine a lower boundary on the channel quality experienced via the use of Master and Slave APs combined with the simplest error correction i.e. retransmitting each packet. One can notice from the results presented that the entire multicast group achieved an improvement in the PER experienced, even those nodes which were located close to the master AP such as nodes 5, 6, 7, 8. Nodes 5, 6, and 7 experienced an improvement in the PSNR by an average factor of $12.82 \mathrm{~dB}$ with Node 8 , the node which receives from two Slave AP observed an improvement factor of $4.68 \mathrm{~dB}$. However, even though the farthest nodes from the Master AP observed a significant decrease in throughput, Node 16 and 17 did not observe enough improvement to obtain an acceptable PSNR. If one were to use the least robust MCS, one should consider a smaller coverage area than the one considered here of $60 \mathrm{~m}$, and hence the nodes at the border between the sectors that each Slave AP is
TABLE V. PACKET REDUNDANCY - MCS-7

\begin{tabular}{|c|c|c|c|c|c|c|}
\hline \multirow[b]{2}{*}{ Node } & \multicolumn{3}{|c|}{ Master AP only } & \multicolumn{3}{|c|}{$\begin{array}{c}\text { Master AP and Slave AP } \\
\text { alternating }\end{array}$} \\
\hline & $\begin{array}{c}\text { PER } \\
(\%)\end{array}$ & Count & $\begin{array}{c}\text { PSNR } \\
\text { (dB) }\end{array}$ & $\begin{array}{c}\text { PER } \\
(\%)\end{array}$ & Count & $\begin{aligned} \text { PSNR } \\
(\mathrm{dB})\end{aligned}$ \\
\hline 1 & 0.04 & 13 & 45.82 & 0.00 & 1 & 41.55 \\
\hline 2 & 0.03 & 13 & 49.37 & 0.01 & 4 & 49.03 \\
\hline 3 & 0.05 & 14 & 46.84 & 0.04 & 9 & 44.19 \\
\hline 4 & 0.07 & 17 & 43.65 & 0.04 & 9 & 45.72 \\
\hline 5 & 2.66 & 25 & 28.85 & 0.11 & 18 & 43.4 \\
\hline 6 & 2.58 & 25 & 28.82 & 0.23 & 24 & 40.32 \\
\hline 7 & 2.84 & 25 & 28.47 & 0.28 & 23 & 40.88 \\
\hline 8 & 2.75 & 25 & 28.88 & 0.88 & 25 & 33.56 \\
\hline 9 & 5.18 & 25 & 26.03 & 0.01 & 3 & 51.64 \\
\hline 10 & 5.76 & 25 & 25.56 & 0.05 & 8 & 42.71 \\
\hline 11 & 5.68 & 25 & 25.68 & 0.35 & 24 & 38.12 \\
\hline 12 & 5.61 & 25 & 25.79 & 1.34 & 25 & 31.83 \\
\hline 13 & 41.10 & 25 & 19.45 & 0.00 & 0 & \\
\hline 14 & 41.10 & 25 & 19.47 & 0.02 & 7 & 47.47 \\
\hline 15 & 41.87 & 25 & 19.36 & 2.82 & 25 & 28.48 \\
\hline 16 & 40.55 & 25 & 19.41 & 11.25 & 25 & 23.36 \\
\hline 17 & 37.13 & 25 & 19.64 & 0.05 & 7 & 43.99 \\
\hline 18 & 22.13 & 25 & 21.11 & 0.02 & 8 & 47.99 \\
\hline 19 & 17.88 & 25 & 21.79 & 0.03 & 5 & 43.81 \\
\hline 20 & 7.38 & 25 & 24.76 & 0.01 & 4 & 48.86 \\
\hline 21 & 39.39 & 25 & 19.41 & 1.53 & 25 & 31.02 \\
\hline 22 & 20.62 & 25 & 21.15 & 0.83 & 25 & 35.13 \\
\hline 23 & 5.04 & 25 & 26.25 & 0.23 & 22 & 42.62 \\
\hline 24 & 3.88 & 25 & 27.19 & 0.20 & 21 & 40.57 \\
\hline 25 & 41.15 & 25 & 19.42 & 3.38 & 25 & 27.65 \\
\hline 26 & 15.56 & 25 & 22.13 & 1.15 & 25 & 32.62 \\
\hline 27 & 4.03 & 25 & 27.24 & 0.36 & 25 & 38.78 \\
\hline 28 & 0.45 & 25 & 36.51 & 0.03 & 6 & 42.81 \\
\hline
\end{tabular}

responsible for are not as far from the Slave AP as the situation considered here.

\section{CONCLUSION}

The results presented in this paper confirm that multiple transmit sources such as APs can be placed at the edge of a coverage area in order to aid those nodes which are suffering the most from the effect of attenuation due to distance from the Master AP. Spatial Diversity on its own can reduce the PER but not enough to achieve good H.264 encoded video, hence a simple error correction scheme, where the Slave AP retransmits every packet is presented. While this is beneficial for MCS-5 and MCS-6, and results in every node achieving a high PSNR, it is not ideal since for these MCS a lower amount of redundancy is required.

Instead of retransmitting the data packets received the next step is to study the effect on PER and video quality when the Slave APs transmit only parity packets. In this manner, the constraint that no changes are applied at the receiver except at the Application Layer, which can be easily modified, is respected. Moreover, it can be highlighted that the restriction imposed on multicast by the IEEE 802.11 standard i.e. no RTS/CTS and no ACK transmitted from the receiver are still maintained. 


\section{ACKNOWLEDGMENT}

The authors would like to acknowledge IST project IST2000-30148 I-METRA for the Matlab Code which simulates IEEE 802.11n channels.

\section{REFERENCES}

[1] "IEEE Std. 802.11 ${ }^{\text {TM }}$-2009, Part 11: Wireless LAN Medium Access Control (MAC) and Physical Layer (PHY) Specifications, Amendment 5: Enhancements for Higher Throughput," 2009.

[2] M. Heusse, F. Rousseau, G. Berger-Sabbatel, and A. Duda, "Performance anomaly of $802.11 \mathrm{~b}$," in Proc. IEEE INFOCOM'03, Apr. 2003, pp. 836-843.

[3] D. Dujovne and T. Turletti, "Multicast in 802.11 WLANs: an experimental study," in Proc. MSWiM'06, Oct. 2006, pp. 130-138.

[4] J.-M. Vella and S. Zammit, "Infrastructure-dependent wireless multicast over 802.11n WLAN," in Proc. SoftCOM'11, Sept. 2011, pp.1-5.

[5] J.-M. Vella and S. Zammit, "Packet losses of multicast over $802.11 \mathrm{n}$ heterogeneous wireless local area network," in Proc. IWSSIP'12, Apr. 2012, pp. 300-303.

[6] J.-M. Vella and S. Zammit, "An analytical study of infrastructuredependent wireless multicast over IEEE 802.11n WLANs," IEEE Trans. Wireless Commun., submitted for publication.

[7] J. M. Vella and S. Zammit, "A survey of multicasting over wireless access Networks," IEEE Commun. Surveys Tuts., accepted for publication.

[8] Ö. Alay, T. Korakis, Y. Wang, E. Erkip, and S. Panwar, "Layered wireless video multicast using omni-directional relays," in Proc. IEEE ICASSP'08, Apr. 2008, pp. 2149-2152.

[9] Ö. Alay, T. Korakis, Y. Wang, and S. Panwar, "Layered wireless video multicast using directional relays," in Proc. ICIP'08, Oct 20008, pp.2020-2023.

[10] Ö. Alay, P. Liu, Z. Guo, L. Wang, Y. Wang, E. Erkip and S. Panwar, "Cooperative layered video multicast using randomized distributed space time codes," in Proc. IEEE INFOCOM'09, Apr. 2009, pp.176-181.

[11] K. J. Yoon, T. S. Kim and Y.Y. Kim, "A novel multicasting scheme over wireless LAN systems by using relay," in Proc. Emerging Directions in Embedded and Ubiquitous Computing, vol. 4097, pp. 550-559, Aug. 2006.

[12] P. Fan, C. Zhi, C. Wei and K. B. Letaief, "Reliable relay assisted wireless multicast using network coding," IEEE J. Sel. Areas Commun., vol. 27, no. 5, pp. 749-762, Jun 2009.

[13] A. Miu, G. Tan, H. Balakrishnan and J. Apostolopoulos, "Divert: finegrained path selection for wireless LANs" in Proc. the ACM Mobisy'04, Jun. 2004, pp. 203-216.
[14] Y. Zhu, Q. Zhang, Z. Niu and J. Zhu, "Leveraging multi-AP diversity for transmission resilience in wireless networks: architecture and performance analysis," IEEE Trans. Wireless Commun., vol. 8, no. 10, pp. 5030-5040, Oct, 2009.

[15] J. Nicholas Laneman, D. N. C. Tse, and G. W. Wornell, "Cooperative diversity in wireless networks: efficient protocols and outage behaviour," IEEE Transactions on Information Theory, vol. 50, no. 12, pp. 3062-3080, Dec. 2004.

[16] A. Adinoyi and H. Yanikomeroglu, "Cooperative relaying in multiantenna fixed relay networks," IEEE Trans. Wireless Commun., vol. 6, no. 2, Feb. 2007, pp. 533-544.

[17] H. Rahul, H. Hassanieh and D. Katabi, "SourceSync: a distributed wireless architecture for exploiting sender diversity," in Proc. ACM SIGCOMM'10, pp. 171-182.

[18] "The ns-3 Network Simulator," [Online]. Available: http://www.nsnam.org/.

[19] J. P. Kermoal, L. Schumacher, K. Pedersen, P. Mogensen, and F. Frederiksen, "A stochastic MIMO radio channel model with experimental validation," IEEE J. Sel. Areas Commun., vol. 20, no. 6. pp. 1211-1226, Aug. 2002.

[20] V. Erceg et al., "TGn channel models," IEEE 802.11-03/940r04.

[21] Q. Xia, M. Hamdi, and K. B. Letaief, "Open-Loop link adaptation for next-generation IEEE 802.11n wireless networks," IEEE Trans. Veh. Technol., vol. 58, no.7, pp.3713-3725, Sept. 2009.

[22] B. Bjerke, J. Ketchum, R. Walton, S. Nanda, I. Medvedev, M. Wallace, and S. Howard, "Packet error probability prediction for system level simulations of MIMO-OFDM based 802.11n WLANs," in Proc. IEEE ICC, May 2005, pp. 2538-2542.

[23] "Football Sequence," [Online]. Available: http://media.xiph.org/video/derf/.

[24] "ffmpeg-0.11," [Online]. Available: http://ffmpeg.org/.

[25] G. Haßlinger and O. Hohfeld, "The Gilbert-Elliot model for packet loss in real time services on the internet," in Proc. $14^{\text {th }}$ GI/ITG Conference $M M B^{\prime} 08$, Apr. 2008, pp.1-15.

[26] M. A. Ismail, G. Piro, L. A. Grieco and T. Turletti, "An improved IEEE 802.16 WiMAX module for the ns-3 simulator," in Proc. SIMUTools'10, Mar. 2010.

[27] Y, Park, Y. Seok, N. Choi, Y. Choi and J-M. Bonnin, "Rate-adaptive multimedia multicasting over IEEE 802.11 wireless LANs," in Proc. IEEE CCNC'06, Jan 2006, pp. 178-182.

[28] S. Zammit, "Digital video transmission over wireless access networks", Ph.D. dissertation, Aston University, 1996. 